\title{
Nucleonic Radiation Gage Control
}

\author{
A British steel mill is using gamma radiation from radio- \\ active isotopes for gage control in a continuous hot strip mill. \\ While X-ray gages are in use in a number of continuous strip \\ mills, gamma radiation appears to offer certain advantages.
}

\begin{abstract}
$I^{\mathrm{N}}$ $\mathrm{N}$ recent years a number of noncontact gages have been developed for testing the thickness of steel in hot and cold strip mills. The majority of tnese are radiation gages, consisting of an ionizing radiation source-radioactive isotopes or an X-ray tube-and a detector responsive to ionizing radiation, together with associated amplifier and recorder. The strip or sheet to be gaged passes between the source and detector; the intensity of radiation falling on the detector varies with the steel thickness, causing the detector output to vary. These variations indicate variations in gage thickness.

Radiation gages with $\beta$ rays are used in industry, and X-ray gages are in wide use on cold strip mills. But $\gamma$ ray gages, the youngest member of this family, appear to offer additional advantages, particularly on hot strip mills producing narrow strip. $\beta$ rays can be used only on strip up to 0.02 in. thick, $\mathrm{X}$-rays up to 0.25 in., but $\gamma$ rays can be used on steel up to $1.5 \mathrm{in}$. thick. Other advantages are: lower cost, less vulnerablity than X-ray equipment, no need for source stabilization, since a $\gamma$ source is absolutely stable over short periods, and there is no need for cooling.
\end{abstract}

\section{First gamma gage at United Steel Co.}

The existing nucleonic thickness gage, made by the Baldwin Instrument Co., has been installed on a Morgan strip mill rolling up to 9 -in. wide strip. It is arranged to measure thickness between the limits of 0.03 and 0.25 in. The tolerance limit can be set on the instrument to suit the rolling specification, which is usually about 0.002 to $0.010 \mathrm{in}$.

Measurement is provided by a strontium $90 \mathrm{ra}-$ dioactive source and a radiation detector placed on either side of the hot strip. The variations in the detector output, after passing through electronic equipment, are shown on a lamp display board and on a teleprinter.

The lamp display board provides a pictorial presentation of the longitudinal profile of the strip. The board has 48 vertical rows of 5 colored lamps. The middle lamps indicate that strip thickness is well within the specified limits; the rows on either side signify that it is just inside of the limits; and the outer rows indicate that it is beyond the tolerance limits. Each vertical lamp row represents the mean thickness of a length of strip, equal to that which passes through the gage in a 1-sec integrating period. Thus, the longitudinal thickness profile of the whole strip is displayed on the lamp board on completion of rolling. A teleprinter is used to provide a permanent record, in digital form, of the longitudinal profile of each strip.

\section{Automatic standardizing}

The source unit of the $\gamma$ gage is fitted with standardizing equipment which is brought into operation automatically between the rolling of successive pieces of strip. The sudden increase in radiation falling on the detector at these intervals changes the instrument from the measuring to the standardizing condition, and a standard piece of steel is interposed between the source and the detector. A servo-mechanism immediately recalibrates and corrects any drift of the instrument. With the arrival of the next strip there is a sudden decrease in radiation falling on the detector which causes the instrument to be restored to the measuring condition.

\section{The detector unit}

Thallium-activated sodium iodide crystals are used as a radiation sensitive element in the detector unit. These crystals scintillate when radiation falls upon them, and the visible light from the crystal is picked up by two photomultipliers. One acts as the measuring device and the other as a relay for the changeover from measuring to standardizing. The measuring photomultiplier feeds an integrating circuit which averages the current over the time cycle of $1 \mathrm{sec}$. The integrator feeds current to the amplifier in the electronic unit which in turn feeds the discriminator. The discriminator classifies each signal into one of five levels depending upon the magnitude of the signal. This in turn depends on the average thickness of strip passing through the measuring instrument during the appropriate second of time. These five levels correspond to those on the display board and the teleprinter.

This comparatively simple and much less expensive method of measuring the thickness of hot steel strip has eliminated fragile tubes, high operating voltages, and associated equipment. Successful operation, as has been demonstrated, should prompt further installations on narrow hot strip mills.

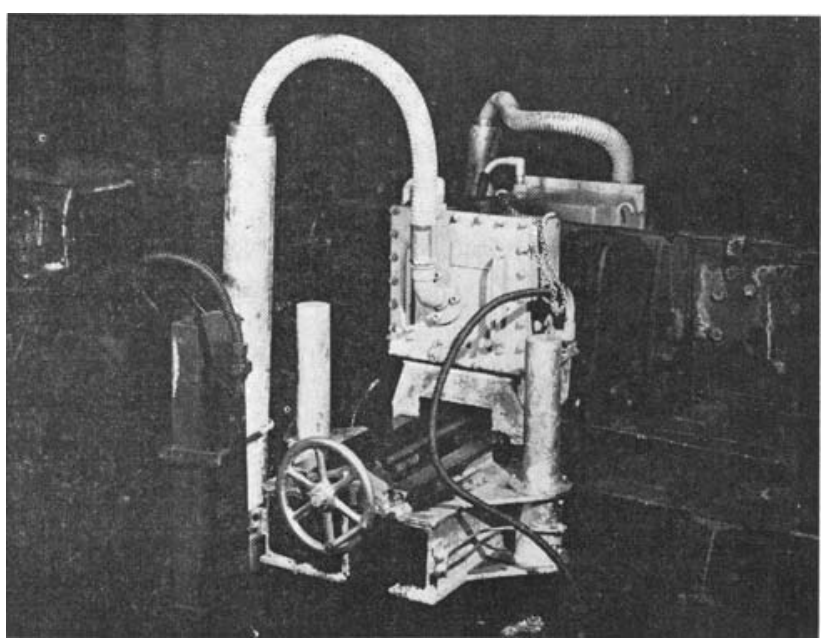

Source holder (in foreground) and detector unit in position on the mill run-out table. 